\title{
People's Attitudes Towards the Use of Robots in the Social Services: A Multilevel Analysis Using Eurobarometer Data
}

\author{
Marco Carradore ${ }^{1}$ D \\ Accepted: 10 September 2021 / Published online: 18 October 2021 \\ (c) The Author(s) 2021
}

\begin{abstract}
Robots have been employed in the industrial sectors for over half a century; however, their appearance in the domestic sphere is a modern phenomenon, occurring in just the last decade. These so-called social robots are carrying out a variety of tasks traditionally carried out by humans, and in contexts in which they must interact with human beings. These kinds of social robots are now being used in the welfare services, providing assistive services and companionship for the infirm or elderly, and even children. Thus, the use of social robots in everyday life has triggered an animated debate about the acceptance of these devices by their end users. In this paper, multilevel analysis is applied using data from the Eurobarometer survey (sample size 27,901, covering 28 countries) to investigate how socio-demographic characteristics and country-level indicators of technological and economic development (the rate of high-technology manufactured exports, the cellular phone subscriptions rate and GDP) influence how robots are accepted in the realm of the social services. The results show that only individual (socio-demographic) and technology acceptance model (TAM) factors influence attitudes towards social robots. The effects of the contextual variables considered were not statistically strong enough to explain the attitudes towards social robots for social services.
\end{abstract}

Keywords Social robot $\cdot$ Social assistance $\cdot$ Europe $\cdot$ Multilevel analysis

\section{Introduction}

Over recent years, we have witnessed an increase in the application of technology in all areas of daily life, and one of the most relevant changes concerns the development of robotic technologies for everyday life activities. Robots are increasingly being used in the healthcare sector (e.g. medical devices) [1,2], and their appearance is also expanding in the entertainment sector (e.g. toys) [3] as well as in the domestic sphere [4-7], where they are used to perform housework tasks, and even social assistance for the elderly or children $[8,9]$.

The development (or "evolution") of robots has many implications for a number of social dimensions, such as welfare policies [10, 11] and legislation [12, 13], as well as from an ethical standpoint $[14,15]$. Their introduction into the welfare and healthcare services has necessitated many

Marco Carradore

marco.carradore@univr.it

1 University of Verona: Università degli Studi di Verona, Verona, Italy new scientific studies, such as those investigating people's attitudes towards robots [17-19]. Trying to anticipate how people may react to new technologies is fundamental because it advances the debate on the issues that concern the potential replacement of human workers with robots [20]. Such data is useful for professional figures, such as policymakers, legislators, programmers, IT experts and those responsible for developing IT education programmes [17], as well as advancing sociological knowledge that aims to help people enhance their health and quality of life.

In summary, this study confirms the impact of certain socio-demographic features, such as gender and living area, on attitudes towards social robots. The extent to which individuals use the internet-a dimension not considered in previous studies-also appears to be correlate with more positive attitudes towards robots. The strong and weak aspects of this research and issues to be addressed by future research are discussed. 


\section{Background}

Over the last decade, a transition in robot use has occurred. Robots are no longer confined to the industrial sector, but have become commonplace in the field of medical surgical devices, and they are also expanding into the domestic sphere and social services [4-6]. Indeed, robots are increasingly found providing social assistance (i.e. companionships) in hospitals, home contexts or other settings for the elderly, disabled or even children $[8,9]$. Although the transition of a robotic device from the industrial into the social sphere might be considered a normal consequence of technological development, the implications of such a transition are highly relevant, not only in terms of legislation $[12,13]$ and welfare policies [10], but also from the ethical standpoint [15]. This is because the transformation in question necessitates that people interact with non-human beings, or are exposed to interactions between non-human beings.

Robots have a long tradition of being used in the industrial and military sectors; these sectors are typically circumscribed in that the actions performed by robots are highly formalized, according to certain task rules, and access to the robots is generally restricted to the individuals (factory workers and military personnel) directly involved in those activities $[4,6,21]$. From a sociological point of view, robots were introduced into manufacturing for three different reasons: (1) to advance the quality of the assembly process; (2) to guarantee the regularity of the production process; and (3) to perform the most dangerous tasks and reduce risk exposure to humans [6]. In the industrial/military context, actions are highly formalized, meaning that human workers and robots must perform specific tasks. Although trade unions and labour movements that aimed to defend employers' rights fought against the introduction of robots into the workplace, robots are now commonplace in numerous industrial sectors [4, 6], and indeed outnumber human employees in some manufacturing settings.

Progress in technology, and an increase in the application of artificial intelligence (AI), has transformed robot shapes and functions, even mimicking biological forms, generating robots increasingly able to interact and communicate with other autonomous physical agents that abide by human social rules, as well as humans themselves [22]. Robots that combine technical aspects with social aspects are the so-called social robots [23] or socially assistive robots [24]. In other words, to be social, a robot needs to be endowed with social functions that allow it to interact according to the context, and it must have a form that explicitly allows it to be social, independent of the end user. Furthermore, these robots must not pose a danger to humans. Assuming this perspective, social robots can be defined as: “... embodied agents that are part of a heterogeneous group: a society of robots or humans. They are able to recognize each other and engage in social interaction. They possess histories (perceive and interpret the world in terms of their own experience), and they explicitly communicate with and learn from interaction" [25], p. 1482].

With the introduction of robots into the domestic sphere of everyday life — or the reproduction sphere, as defined by Fortunati [22], but see also Fortunati, Esposito et al. [4] and Taipale et al. [6] - social robots have started to operate in an environment that is much less formalized than that of the industrial or surgical sector. They have to carry out tasks, such as providing companionship, assisting elderly or disabled people - work that is usually performed by women and is low paid [4], and within a context where no organized social movements exist that fight to defend the interests and rights of those who will be exposed to the robots, i.e. the end consumers of these engineered products. Indeed, we might say that social robots are being required to simulate human beings [26].

There are many examples in the literature of the use of social robots for performing clinical procedures, thus in the field of medical services (for reviews on this topic see: Chen et al. [11]; Leite et al. [27]; Wang et al. [28]), whereas little research has been made into the use of social robots in the social services, such as for the provision of companionship. This division in social robot functions between the medical and social spheres reflects two very different types of application, as underlined by Coghlan [29]: functional care (e.g. monitoring physiology, diagnosing and lifting), and affective care (e.g. comforting, bringing enjoyment to isolated people and stimulating emotions). An example of the latter is Sony's robot dog AIBO, which can be used in care setting as well as at home for people in need of companionship [29].

The adaptation of the robot for social or affective care has given rise to new scientific questions, not only from the technological perspective, but also from a sociological viewpoint; examples include: what are people's attitudes towards affective robots, and what are the individual and social dimensions that determine acceptance towards social or assistive robots? To form hypotheses on the potential consequences of their introduction, and to provide data useful for those responsible for developing IT education programmes and policies regarding robot use (policymakers, programmers, IT experts and educators), it is of great importance that we first attempt to answer these questions.

One of the most applied theoretical models used to identify the factors that predict people's motivations for adopting new technologies is the technology acceptance model (TAM) [30]. Grounded in the theory of reasoned action (TRA) [31] and in the theory of planned behaviour (TPB) [32], TAM was originally used to assess the general acceptance of computers, but since the rapid expansion of other novel technologies, many researchers continue to turn to this model to explain the human acceptance of these innovative tools. Furthermore, the number of the predictive variables included in the TAM 
model has grown over time. The key indicators applied in the original model included: perceived usefulness (PU), and perceived ease of use (PEOU) [33]; whereas, examples of those added later on include: compatibility, privacy, normative beliefs, and self- efficacy used to explain consumer intention to use on-line shopping [34], trust in technology used to predict people's intention to use smart-city services [35], and anxiety or technophobia with regard to predicting the public perception of AI/robot-related technologies [33]. TAM was further expanded recently by adding variables that concern the contextual level [33].

\subsection{Attitudes towards Robots in Europe}

The Eurobarometer surveys provide the main source of data on people's attitudes towards robots in Europe. These surveys have been monitoring the acceptance of robots for performing certain tasks in various fields of application since the 2012.

Taipale et al. [6], using data from the Special Eurobarometer 382 , pertaining to the year 2012, and applying ordinal logistic regression, investigated the socio-demographic profiles of European citizens already exposed to robots in social scenarios. From their analysis it emerged that "large cities seem to be the most favourable places for the introduction of robots into the health and care sectors. While in villages and smaller towns attitudes towards the care and health services seem to be still family and community oriented, in the large cities people are used to relying more on external or technological aid" [6], p. 22]. Furthermore, the results showed that pensioners are not reluctant to use robots in the sphere of domestic life, but a concern does persist regarding reduced human contact; however, this result may was probably due to the misrepresentation of robots, which were seen as substitutes for human beings.

Moreover, Hudson et al. [18] analyzed people's attitudes towards robots used in caring for the elderly. What the researchers found is that, compared with young people, the elderly were more hostile towards the use of robots. Women supported the use social robots less than men, and those who live in larger town and cities expressed more confident attitudes towards robots compared with those from villages and small towns. Although the analyses carried out by Taipale and colleagues [6] and by Hudson et al. [18] are two of the few explicative analyses to assess robot acceptance, they did not consider the role of context [36].

The importance of including the effect of context in the analysis of the acceptability of robots has been underlined Katz et al. [37]. In their analysis of human-robot interactions, they highlighted that the cultural differences between groups are relevant for robot acceptance. The same conclusion was drawn by Li et al. [38], who stated "we observed significant interaction between culture and robot task and tend to conclude that when the tasks are mainly differentiated by the robot's utterances, people from a low-context culture are more influenced in the emergent scale than people from a high-context culture are" [33], p. 184].

The environmental effect was, however, considered by: Turja and Oksanen [39], who focussed more on attitudes towards robots in the workplace; Gnambs and Appel [17], ${ }^{1}$ who also contemplated how attitudes towards robots have changed over the years in the $28 \mathrm{EU}$ countries; and Vu and Lim [33], who studied the effects of individual and country factors on public acceptance of artificial intelligence and robotic technologies.

Turja and Oksanen [39] explored the concept of robot acceptance at work, applying TAM in a cross-national comparison study. They used a multilevel method that also allows the context effect to be considered in the analysis. The main results obtained at the individual level were that more robot acceptance is shown by males, highly educated people, the unemployed and citizens with previous experience of robots; at the country level, robot acceptance was more diffuse in nations with a high level of IT (information and technology) exports, a high diffusion of cellular phones and a low risk of job-automation. In conclusion, as stated by the authors, the national-level attribute contributes to the explanation of the total variance. However, it is important to remember that in the Turja and Oksanen [39] study, the context considered regarded the acceptance of robot assistance in a manufacturing context.

Gnambs and Appel [17], using Eurobarometer data from three different years (2012, 2014 and 2017), applied a multilevel analysis to investigate changes in attitudes over time, as well as individual- and country-level differences. The individual features they considered were: gender, age, years of education and employment; whereas the country characteristics they considered were: percentage of older citizens, unemployment rate, percentage of technology export, research investment and geographical latitude and longitude. The focus of their research was more on the general appraisal of robots, measured as a positive or negative view of robots in combination with the following two statements: robots are good for society; and robots are needed to do jobs that are hard for people to do. In line with previous studies, Gnambs and Appel [17] found that men-who are more likely to have a higher education level than women-have more favourable attitudes towards social robots compared with woman. With regard to employment conditions, Gnambs and Appel [17] distinguished between whiteand blue-collar workers and the unemployment, and they discovered that white collar workers were associated with slightly more positive attitudes towards robots than blue collar workers and the unemployed. In contrast with other

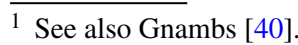


studies (e.g. Hudson et al. [18]), they found age to have a negligible impact on the evaluation of robots. Regarding contextual variables, the authors identified that societies characterized by a high proportion of elderly people were more inclined to have positive attitudes towards robots, higher unemployment rates corresponded with a very low level of positive attitudes towards robots, and northern countries showed significantly more positive attitudes towards robots than countries located in the south. Although the authors also considered task-specific attitudes towards robots, which included helping the elderly and infirm, they did not offer any in-depth discussion on this specific topic-for example, by considering the effect of national context-leaving this area as yet unexplored.

$\mathrm{Vu}$ and Lim [33], using Eurobarometer 87.1 data, investigated the factors influencing public attitudes towards the acceptance of $\mathrm{AI} /$ robots. The three latent variables-digital technology efficacy, perceived threat of general job loss and usefulness - and the single-question variable, prior knowledge of AI (but these two lasts were excluded in the final model, as some demographics variable), constitute the individual-level factors that were combined with three national level variables-innovation, government effectiveness and GDP per capital-in a multilevel SEM analysis. The results, in addition to proving the relevance of individual factors in shaping public attitudes towards AI/robotics, support that TAM should include the technophobia dimension in addition to the two traditional variables (perceived usefulness and perceived ease of use). In fact, the perceived threat of general job loss was found to have a significant negative impact on the acceptance of $\mathrm{AI} /$ robots, while digital technology efficacy positively impacts the attitudes towards of $\mathrm{AI} /$ robots. At the country level, however, it emerged that the techno-socio environment has not had any direct significant impact on the acceptance of $\mathrm{AI} /$ robots.

\subsection{Research Hypotheses}

To date, social research into robots has mostly investigated attitudes towards robots in general and addressed people's concerns about their use in the workplace. The use of robots for performing specific social tasks, such as providing companionship, has received much less attention; thus, considering their growing role in this domain, it is crucial that we advance our knowledge about the factors at both the individual and contextual levels that may influence people's attitudes towards social robots for social services.

To address this gap in the literature, I applied the TAM approach, as proposed by Vu and Lim [33], which takes many more socio-demographic (i.e. individual) features, not previously considered in the past literature, into consideration. In addition to the individual variables traditionally considered, such as gender, age, education and area of living, this analy- sis also included family condition, the number of children in the family and social class to investigate whether these features influence people's attitudes towards robots for social services.

Based on our current knowledge of robot acceptance, I propose the following hypotheses:

H1 Perceptions about the use of robots in the social services vary between different European countries (as suggested by previous studies considering both individual and contextual level variables).

H2 (Hypotheses concerning individual-level predictors) older women with a low education level and living in rural areas are less likely to have a positive attitude towards social robots, whereas belonging to a higher social class could positively influence robot perceptions (H2a). A high number of the children in the family may negatively influence attitudes towards social robots, while being a worker may positively affect the dependent variable (H2b). Moreover, I postulate that technical knowledge (such as knowledge about artificial intelligence) in individuals and the two TAM predictors (digital technology efficacy and perceived usefulness) positively influence attitudes towards robots, whereas the perceived threat of general job loss is likely to impact attitudes in a negative manner $(\mathrm{H} 2 \mathrm{c})$. Finally, I hypothesize that people who use the internet frequently are more likely to express positive opinions about robots ( $\mathrm{H} 2 \mathrm{~d})$.

H3 Since the level of AI knowledge varies between countries, I postulate that the effect of AI knowledge on attitudes towards social robots is stronger in some countries than in others.

H4 Although the rate of high-technology exports (an indicator of a country's technological development) was previously found to have a discordant effect on attitudes towards social robots [17, 39], I postulate that a high level of technological development, a high cellular phone subscription rate, and a high level of economic development are likely to correlate positively with attitudes towards social robots.

H5 Finally, the relationship between attitudes towards social robots and AI knowledge may vary as a function of (or, be moderated by) the degree of technological development of the country.

\section{Data and Methods}

\subsection{Data}

The data used in this analysis come from the Eurobarometer surveys. The surveys were founded by the EU and are administered by the European Commission. The Eurobarometer 
data are collected by means of face-to-face interviews at the respondents' homes and they are conducted in the mothertongue language of the participants. Each survey involves approximately 1000 interviews per country of the population aged 15 years or over. Sampling involves the random selection of a sampling point after stratification according to the distribution of the national and regional population. The Eurobarometer database is an open source, and data can be downloaded from the various European data archives (e.g. GESIS-Leibniz-Institute for the Social Sciences ${ }^{2}$ and EU Open Data Portal $\left.{ }^{3}\right)$.

Three different Eurobarometer surveys have investigated topics regarding social robots: Eurobarometer 77.1 (year 2012) ${ }^{4}$; Eurobarometer 82.4 (year 2014) ${ }^{5}$; and Eurobarometer 87.1 (year 2017). ${ }^{6}$ The questions used to investigate robot issues were not always the same, and the concept of artificial intelligence was first introduced in the most recent survey. The surveys provide a definition of what is intended by the term robot together with two example photographs. ${ }^{7}$

For the present study, only the most recent survey is used. Its sample is composed of 27,901 residents (level-one) from across the $28 \mathrm{EU}$ member states (level-two).

\subsection{Individual-Level Variables}

The acceptance of robots for social services and companionship — the dependent variable - was measured by a question which asked how the respondent felt about: "having a robot provide you services and companionship when you are infirm or elderly". The response scale ranges from totally uncomfortable (value: 1) to totally comfortable (value: 10) (M $=4.22$, SD = 3.01). The answers "it depends" (spontaneous) and "DK" (don't know) were excluded, as were non responses.

The explicative variables considered include sociodemographic indicators, indicators of use of information and communication systems, the TAM indicators as suggested by $\mathrm{Vu}$ and Lim [33], and an indicator of AI knowledge. Specifically, the sociodemographic indicators are: gender-used in

\footnotetext{
${ }^{2}$ For more information see: https://www.gesis.org/en/home.

${ }^{3}$ For more information see: https://data.europa.eu/euodp/en/home.

${ }^{4}$ https://search.gesis.org/research_data/ZA5597.

5 https://search.gesis.org/research_data/ZA5933.

6 The GESIS Data archive codes are: ZA No. 5597, ZA5933 and ZA6861, respectively.

7 The definition is: "A robot is defined as a machine which can assist humans in everyday tasks without constant guidance or instruction, e.g. as a kind of co-worker helping on the factory floor or as a robot cleaner, or in activities which may be dangerous for humans, like search and rescue in disasters. Robots can come in many shapes or sizes and some may be of human appearance. Traditional kitchen appliances, such as a blender or a coffee maker, are not considered as robots" (Eurobarometer 87.1 questionnaire).
}

the model as a dichotomic female vs male variable (reference category $=$ male). Age, years spent in education and number of children (aged less than 14 years old) in the family-considered as a continuous variable. Employmen$\mathrm{t}$-which indicates the current occupation of the interviewee; responses were recoded as: worker (e.g. farmer, fisherman, professional, shop owner, business proprietors, employed professional), house person (e.g. responsible for ordinary shopping), unemployed, retired and still studying (reference category $=$ worker). Family condition-ranging from single (living with or without children), single living with a partner, married or re-married with or without children (including children from previous marriages/unions), or divorced, separated or widowed; each option possible with or without children. For the analysis of the present study, I considered the following clustered categories: unmarried, (re)married/unmarried with partner, divorced or separated, and widowed (reference category $=$ single with or without children). Status - self-assessed as belonging to one of five class options. The responses were clustered into three categories: lower-which includes the working class and the lower middle class of society; middle class; and upper class-representing the sum of the upper middle and the higher classes of society (reference category $=$ lower). Living location-classified as: rural area or village, small- or middle-sized town, or large town (reference category $=$ rural area or village).

As a measure of the use of information and communication systems, the analysis considered the frequency of the use of the internet (net use). This information was computed by summing the responses to the following questions: use of the internet at home, in the work place, on personal mobile devices (laptops, smartphones, tablets, etc.), or elsewhere (school, university, cyber-café, etc.). Each variable contained 4 response categories that were: every day or almost every day (reference category), two or three times a week, about once a week (options were recoded as: weekly), two or three times a month (options were recoded as: monthly or less), less often or never, and no internet access (recoded as never/no access).

The first indicator of technology experience considered AI knowledge. This was measured by asking respondents whether they had heard, read or seen anything about artificial intelligence in the previous 12 months. The response constituted a dichotomic variable (positive responses $=1$; negative responses $=0$ ) (reference category $=$ positive responses).

The TAM indicators are perceived usefulness, digital technology usefulness and perceived threat of general job loss, all considered as continuous indicators. The single variables used to identify the latent factor are reported in Table 2 . The missing data for each variable were excluded from the analysis. 


\subsection{Country-Level Variables}

The contextual-level variables included in the model were: (i) GDP per capita, as an indicator of economic development $(\mathrm{M}=100.50, \mathrm{SD}=65.78$, range 25.20-324.70); (ii) the high-technology exports rate $(\mathrm{M}=12.039, \mathrm{SD}=7.01$, range 4.30-34.50), which concerns products associated with high $\mathrm{R} \& \mathrm{D}$ intensity, such as aerospace products, computers, pharmaceuticals, scientific instruments and electrical machinery; and (iii) the cellular phone subscriptions rate (per 1000 people) $(\mathrm{M}=123.00, \mathrm{SD}=13.40$, range 99.45-153.28). The first two variables were drawn from the Eurostat database, ${ }^{8}$ whereas the latter was drawn from the World Development Indicators database. ${ }^{9}$ All national-level variables refer to the year 2017.

\subsection{Statistical Techniques}

In order to investigate how micro (individual) and macro (contextual) dimensions affect people's attitudes towards the use of robots for social services, multilevel analysis was carried out. The multilevel model is an extended form of regression analysis that can be used to handle grouped data, such as clusters of individuals [41], schools [42] or other kinds of groups [43]. In this secondary analysis, the first level is formed by the individuals who took part in the survey, whereas the second level is composed of the 28 European member states. ${ }^{10}$

Before applying multilevel analysis, and in accordance with $\mathrm{Vu}$ et al. [33] who identified the structure of the TAM factors using the same database, confirmatory factor analysis $(\mathrm{CFA})^{11}$ was applied to test whether the relationships between the observed variables and their underlying latent construct could be confirmed. Digital technology efficacy was computed using three 4-point-scale items (1: totally disagree; 4: totally agree), whereas the perceived usefulness of robots was assessed using two 4-point-scale statements (1: totally disagree; 4 : totally agree). The perceived threat of general job loss due to robots was computed using two 4point-scale statements (1: totally disagree; 4 : totally agree). All the single variables used to construct these factors are specified in Table 2. Tables 1 and 2 report the results of the CFA.

\footnotetext{
8 https://ec.europa.eu/eurostat/data/database.

9 https://databank.worldbank.org/source/world-developmentindicators.

10 In the paper, the first level concept is used in an interchangeable way with concept level 1, i.e. the 'people' or 'individual' level. In the same way, the second level concept is related to level 2, i.e. the 'country', 'state' or 'community' level, as well as the 'group' level.

11 This was carried out using the 'lavaan' package in R.
}

Table 1 Confirmatory factor analysis (CFA) results

\begin{tabular}{ll}
\hline Lavaan 0.6-9 ended normally after 39 iterations & \\
Estimator & ML \\
Optimization method & NLMINB \\
Number of model parameters & 17 \\
Number of observations & 23,593 \\
Model test user model & \\
Test statistic & 304.554 \\
Degrees of freedom & 11 \\
p-Value (Chi-square) & 0.000 \\
RMSEA & 0.034 \\
SRMR & 0.019 \\
CFI & 0.996 \\
TLI & 0.993
\end{tabular}

For each factor (computed by summing the single variable values weighted by the CFA values), the internal consistency was then checked. Digital technology efficacy: $\mathrm{M}=0.63$, $\mathrm{SD}=5.07$, Cronbach's $\alpha=0.94$; perceived usefulness: $\mathrm{M}$ $=0.85, \mathrm{SD}=5.07$, Cronbach's $\alpha=0.64$; perceived threat of general job loss: $\mathrm{M}=0.81, \mathrm{SD}=2.72$, Cronbach's $\alpha=$ 0.69 .

Multilevel techniques allow us to estimate: (1) fixed parameters which represent the overall relationships between individual factors and robot acceptance across all countries; (2) the random parameters which denote the variations between countries in robot acceptance; and (3) the effect of country-level predictors and the interaction of this effect (also a fixed parameter).

The analyses were carried out using the lmer function (Linear Mixed Effects in R) from the lme4 package. The maximum likelihood method was used as estimator. The model-based approach [41] was followed for the analysis, thus the weights of the variables were not applied. The following models were sequentially developed:

Model 1 Null model: a two-level null (or empty) ${ }^{12}$ model of the individuals (level 1) nested within countries (level 2), with no predictor variables in the fixed and random part of the model. This model can only identify the intercept and any community effects. In this null model, the variation in attitudes towards robots is distributed across individuals (within countries) and between countries.

Model 2 Random intercept model: this is the same as model 1 , but it includes all the individual explanatory predictors in the fixed part of the model. This model assesses the effect of individual predictors on attitudes towards robot acceptance.

\footnotetext{
12 This model is also called the 'empty model' because it does not consider the explanatory variable [36].
} 
Table 2 Full confirmatory factor analysis model

\begin{tabular}{|c|c|c|c|c|}
\hline Factor & Estimate & SE & z-Value & p-Value \\
\hline \multicolumn{5}{|l|}{ Digital technology efficacy } \\
\hline \multicolumn{5}{|l|}{ You consider yourself to be sufficiently skilled } \\
\hline In the use of digital technologies in your daily life & 1.000 & & & \\
\hline $\begin{array}{l}\text { In the use of digital technologies to use online public services, } \\
\text { such as filing a tax declaration or applying for a visa online }\end{array}$ & 1.061 & 0.005 & 205.897 & 0.000 \\
\hline $\begin{array}{l}\text { In the use of digital technologies to benefit from digital and online } \\
\text { learning opportunities }\end{array}$ & 1.047 & 0.005 & 208.526 & 0.000 \\
\hline \multicolumn{5}{|l|}{ Perceived usefulness } \\
\hline $\begin{array}{l}\text { Robots and artificial intelligence are a good thing for society, } \\
\text { because they help people do their jobs or carry out daily tasks at } \\
\text { home }\end{array}$ & 1.000 & & & \\
\hline $\begin{array}{l}\text { Robots are necessary as they can do jobs that are too hard or too } \\
\text { dangerous for people }\end{array}$ & 0.562 & 0.055 & 28.219 & 0.000 \\
\hline \multicolumn{5}{|l|}{ Perceived threat of general job loss } \\
\hline $\begin{array}{l}\text { Due to the use of robots and artificial intelligence, more jobs will } \\
\text { disappear than new jobs will be created }\end{array}$ & 1.000 & & & \\
\hline Robots and artificial intelligence steal people's jobs' & 1.544 & 0.055 & 28.219 & 0.000 \\
\hline
\end{tabular}

Model 3 Random slope model: this model includes the same independent variables as model 2 (the "random intercept model"), but the dichotomized AI knowledge indicator-an individual explanatory predictor-is allowed to vary between countries for the reasons set out in the theoretical framework.

Model 4 Contextual model: in addition to all the individual explanatory indicators, this model includes the effect of the country level variable. Thus, this model assesses the effect of context on attitudes towards robots.

Model 5 Cross-level contextual model: in addition to the previous indicators, this final model computes the interaction between the individual variable-i.e. the dichotomized AI knowledge-and the contextual indicator.

The application of multilevel analysis to the study of technological change has not been widely used; however, as shown by Turja and Oksanen [39], this method presents numerous possibilities, such as the ability to analyze nested data and to handle complex models.

\section{Results}

The results of the statistical analyses for all five models fitted are presented in Table 3. Since the first hypothesis takes into consideration the role of context, the analysis was initiated by fitting the null model (model 1), which does not contain any individual- or country-level characteristics. As underlined by Snijders and Bosker [41], this model helps to decompose the variability in the data between the individual and country levels, and provides a baseline for comparing the size of the contextual variation in attitudes towards robots in the subsequent models $[43,44]$. The null model only incorporated random effects of context in order to model between-country variation in attitudes towards social robots. In this model, the overall mean across countries was estimated as 4.20. ${ }^{13}$

The between-country variance in the attitudes towards social robots was estimated to be 0.52 , whereas the withincountry variance (i.e. between individuals) was 8.63. The country differences are clearly expressed in the caterpillar plot illustrated in Fig. 1. The plot shows the estimated residuals for all 28 countries in the sample. For more than half of the states, the $95 \%$ confidence interval does not overlap the horizontal line at zero, indicating that the overriding attitudes towards social robots for social assistance in these communities were significantly different to the average response (i.e. above or below the zero line). A clear difference at the country level emerged from the analysis of this model.

To identify the proportion of the total variance that is due to between-country differences, the variance partition coefficient (VPC) ${ }^{14}$ was computed. Thus, $5.68\left(\chi^{2}=1173.08\right.$, $\mathrm{p}<0.000)$ of the total variance in the dependent variable (i.e. the acceptance of robots as providers of welfare services) can be attributed to community characteristics (or betweencountry variation). This value is in line with that obtained by previous studies, and denotes that most of the variance

\footnotetext{
13 As stated by Steele "A multilevel model can be thought of as consisting of two components: a fixed part which specifies the relationship between the mean of y and explanatory variables, and a random part that contains the level 1 and 2 residuals" [45]. For this reason, the two components are shown separately in Table 3.

14 The VPC represents the proportion of the total observed individual variation in the outcome that is attributable to between-cluster variation. The higher this proportion, the higher the general contextual effect.
} 


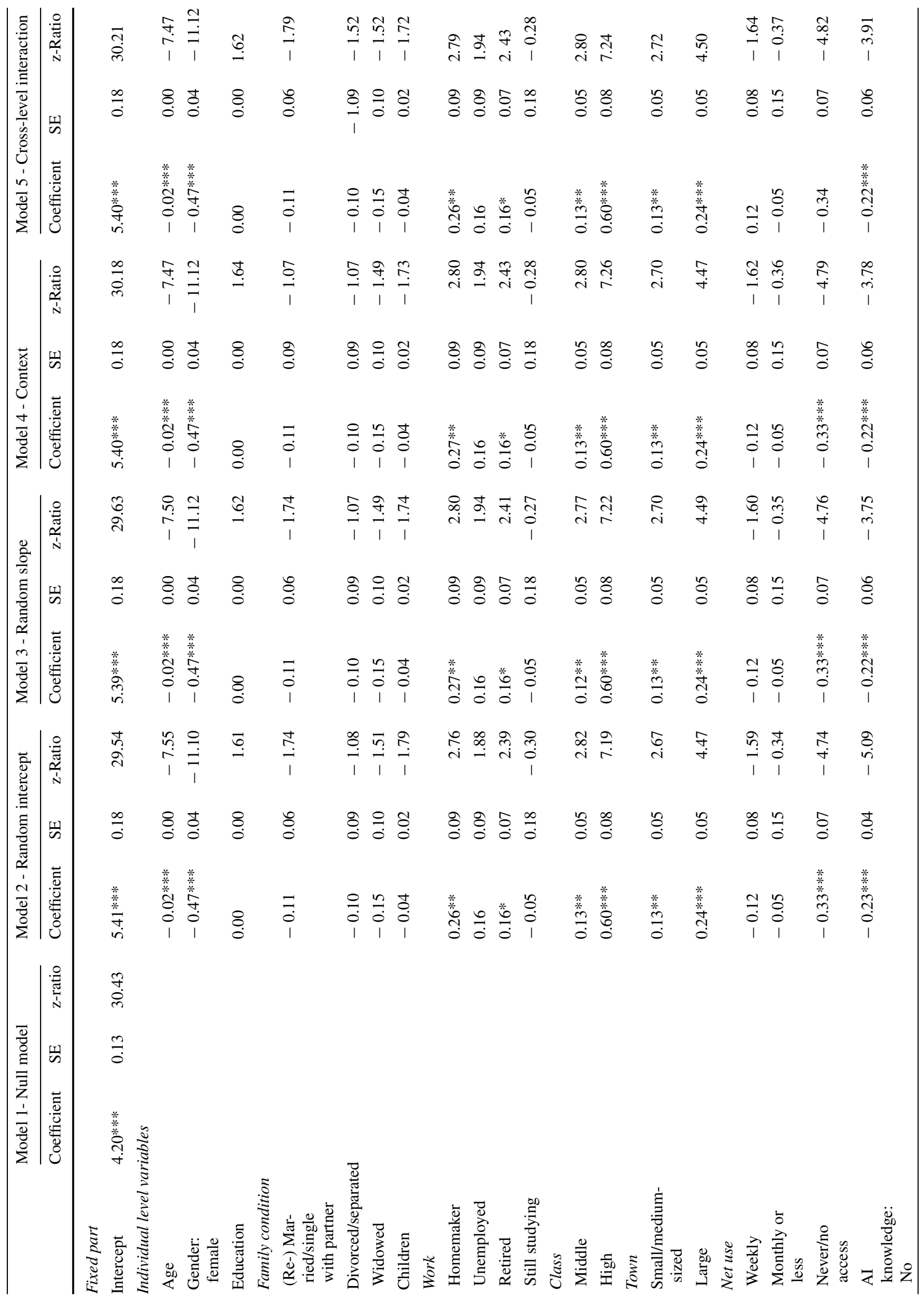




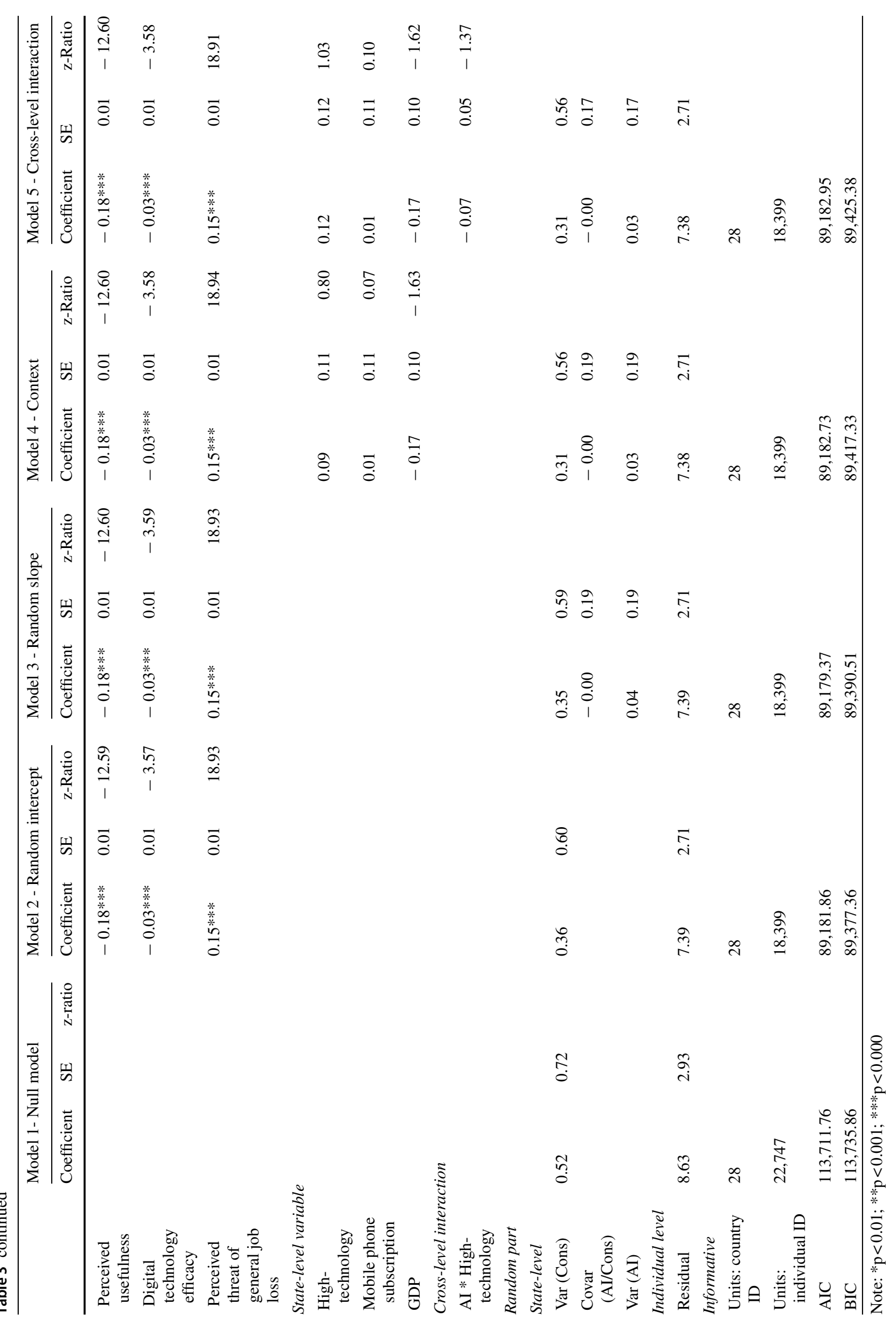




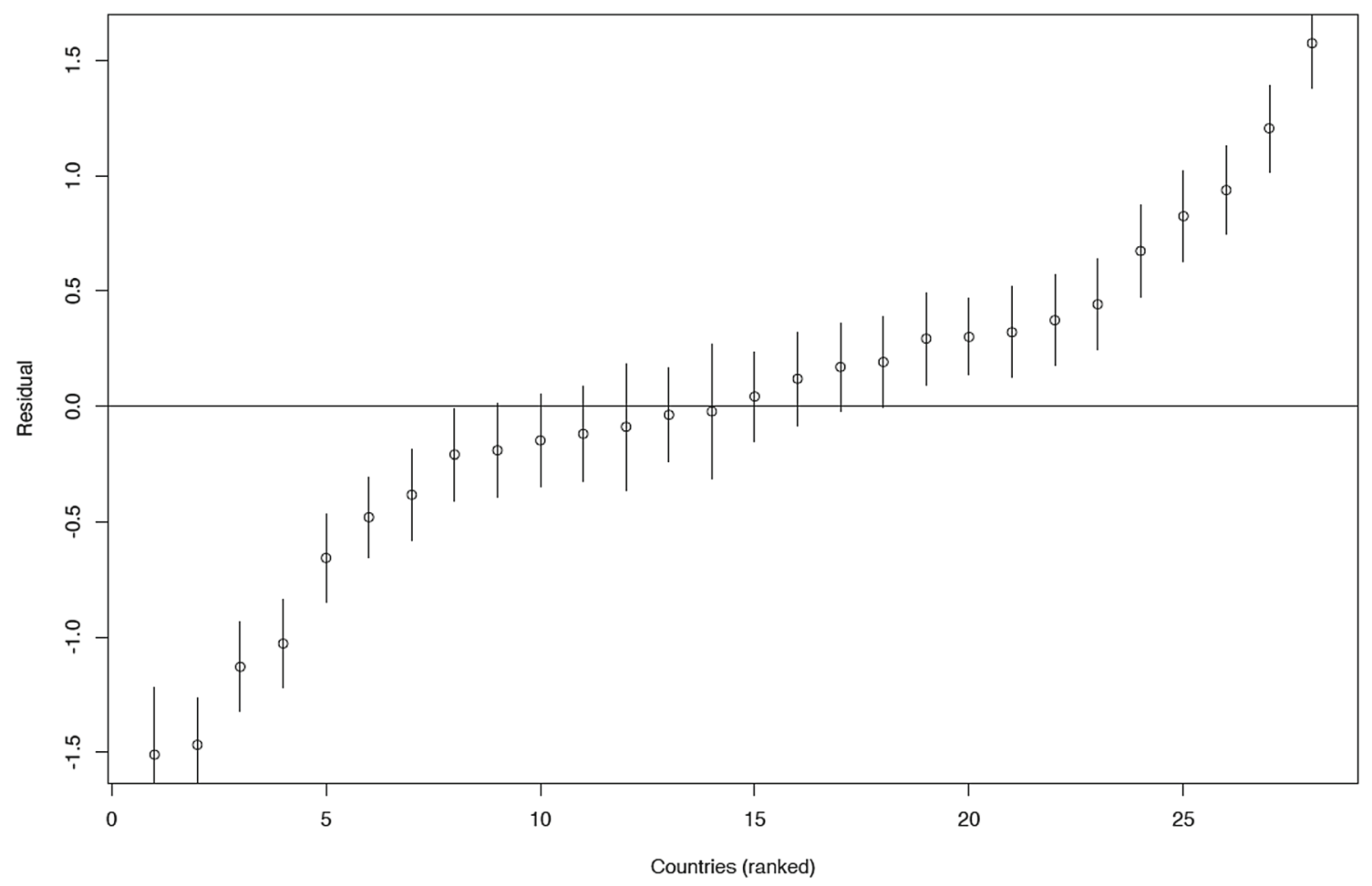

Fig. 1 Caterpillar plot (model 1) showing country residuals and 95\% confidence intervals of attitudes towards social robots

is attributed to differences between respondents, while little variance is due to the context effect. However, although the effect of context on the dependent variable is limited, it is important to take into consideration, and justifies the application of multilevel model analysis.

The impact of the predictive individual factors upon the dependent variable-that allow us to answer the second hypothesis-were included in the random intercept model (Table 3, model 2). This model is based on the assumption that the relationship between the dependent variable and the independent variables is the same for each country, although the intercept (i.e. the average outcome for an individual with a given set of characteristics) varies between the level-2 units [44]. In other words, from this model, we might expect each participant to exhibit some individual differences that affect the intercept, but not the slope. By adding explanatory variables to the random intercept model, we then investigated the extent to which they are able to explain the variability of the dependent variable at both levels (the individual and the country level) [36].

The random intercept model shows that although the effect of age is significant, it has a negligible $(-0.02)$ effect on people's attitudes towards social robots. Whereas being female reduces the acceptance of social robots for companionship by around 0.47 points compared with being male. Being a homemaker significantly increases positive attitudes towards social robots (by around 0.26 points) compared with being a worker. The same significant positive effect is present if we compare being a worker with being retired: being retired increases positive attitudes towards social robots by 0.16 points compared with the reference category. Both the social class and place of living have significant positive effects on the dependent variable. The probability that an individual expresses a positive attitude towards social robots is 0.13 points higher in those who consider themselves to belong to the middle class compared with those rating themselves to pertain to the lower class, and 0.6 points higher compared with those declaring to belong to the higher class. Moving from a rural area to a small/medium size city increases social robot acceptance by 0.13 points, whereas moving from a rural area to a large town increases acceptance by around 0.24 points. Having vs not having access to, or not using the internet, also has a significant impact on social robot acceptance. The probability of accepting robots for companionship is 0.33 points lower in people that never use internet compared with those who use it daily.

The acceptance of social robots in people with no previous AI knowledge is around 0.23 points lower than that for people with AI knowledge.

The TAM variable perceived usefulness has a significant negative effect on people's attitudes towards social robots equivalent to 0.18 points; whereas the variable digital technology efficacy, although having a significant negative impact on the dependent variable, its effect is not relevant. 
Finally, the increase in the perceived threat of general job loss (an anxiety indicator) generates a significant drop by 0.15 points in attitudes towards social robots.

In the random intercept model, variation at the country level was estimated after allowing for, and conditional on, selected individual compositional characteristics. After controlling for individual compositional characteristics, we can see that the variation between countries decreases in magnitude slightly, from 0.52 (model 1) to 0.36 (model 2), thus the VPC becomes $4.64 \%\left(\chi^{2}=725.09, p<0.000\right)$. In other words, this result confirms that context exerts an effect on the variation in the attitudes towards social robots.

The answer to the third hypothesis is derived from the random slope model (model 3), in which the indicator AI knowledge was allowed to vary between countries, whereas the effect of each of the other explanatory variables was assumed to be the same for each country. This was done because we postulated that the relationship between AI knowledge and attitudes towards social robots might differ between individuals in addition to the baseline differences; thus, both the intercept and the slope of the AI knowledge predictor are allowed to vary randomly across countries. ${ }^{15}$ Moreover, as our study entailed the testing of a specific hypothesis, a random slope should theoretically have been selected. However, as stated by Snijders and Bosker [41] "if the theory (whether this is general scientific theory or a practical policy theory) does not give any clue with respect to a random slope for a certain predictor variable, then one may be tempted to refrain from using random slope. However, this implies a risk of an invalid statistical test ... In data exploration, one can try various specifications. Often it appears that the chance of detecting slope variation is high for variables with strong fixed effects" $(2012,87)$. In our case, since no strong theory exists concerning attitudes towards the use of social robots for social assistance, the decision to use a random slope for the indicator AI knowledge was based on my own personal hypothesis and not on an established theory. That said, this choice was in line with the "strong fixed effect criterion" illustrated by Snijders and Bosker [41].

The values of the parameters in the fixed part of model 3 are approximately the same as in the random intercept model. The additional parameters introduced into the random slope model are: the slope variance (since the AI knowledge variable has a random effect), and the intercept-slope covariance.

The intercept-slope covariance estimate was negative, and its effect was negligible (Covar (AI/Cons) $=-0.00)$, thus the relationship of the independent and dependent variables does not differ across countries.

\footnotetext{
15 This process adds new conditions to the model: the slope variance and the covariance between intercept and slope. These new data values are presented in the "random part" section of the "random slope model" (model 3) presented in Table 3.
}

The effect of the contextual variables was tested in the context model (model 4), in which the country-level variables were included in the model in precisely the same way as the people-level variables. Once again, it was possible to notice a significant fixed effect of the individual level predictor, which was of approximately the same value as in the previous models. The three contextual variables considered as possible predictors at the country level of attitudes toward social robots for social services and companionship did not have a significant effect. Thus, considering the non-relevant effect of these context factors, we cannot say that the attitude towards social robots between countries can be accounted for by the country-level characteristics examined in this paper.

The interaction of the AI knowledge with the contextual variable "high-technology export rates" is presented in the cross-level interaction model ${ }^{16}$ (model 5). This model was designed to test the extent to which AI knowledge, moderated by the rates of high-technology context, accounts for attitudes towards social robots. Since the context variable was not significant, it would be worth removing this non-significant variable from the model. However, as suggested by the literature (see, for example, Snijders and Bosker [41]), if a theoretical reason exists to consider the interaction, even if the independent variable is not significant, it is right to applying a multilevel cross-level interaction analysis. In this case, the interaction was computed following the theoretical idea that a technological context influences people's knowledge about AI [46]. In other words, we might postulate that the effect of being in a country characterized by high-technology exports was not significant on average, but could have a positive effect on those who have AI knowledge and on those who do not have AI knowledge. ${ }^{17}$ The coefficient of the interaction was equal to -0.07 and was not significant, thus it is negligible.

For each model, the fit information AIC and BIC statistics were computed. These metrics, used to compare models, permit us to assess whether the fitted models are improved or not by including the independent variables" (the smaller their values, the better model fit). The AIC and BIC statistics are lower when the independent variables are included in the model. Thus, the AIC statistic decreases a little further when AI knowledge is allowed to slope in the mode, whereas the BIC value increases. The chi-square test for deviance was also applied, and this indicated the random-intercept slope model $\left(\chi^{2}=6.4867, p<0.05\right)$ to have the best fit.

\footnotetext{
16 The interaction model offers the opportunity to check whether the effect of one explanatory variable on the dependent variable depends on the value of another explanatory variable.

${ }^{17}$ It was also checked, by means of ANOVA, the relation between the AI knowledge and the high-technology exports indicator and the relation is significant $(F(1,23,242)=59.1, p<0.000)$.
} 


\section{Discussion and Conclusions}

The aim of this study was to expand upon the current body of literature on cross-country variations in perceptions about the use of robots in social services. The multilevel approach was used to test five specific hypotheses.

The results sustain $\mathrm{H} 1$, which predicted the existence of significant between-country variability in attitudes towards social robots. They show that a difference in the attitudes towards robots exists at the country level, although they cannot affirm that this difference depends on high-technology export rates, on mobile phone subscription rates or on the indicator of economic development (i.e. GDP) as hypothesized in H4. Since the contextual indicators analyzed here were not significant, the possibility that environment can affect attitudes towards social robots remains an open issue. As far as context is concerned, in their study Turja and Oksanen [39] found a significant effect of ICT exports, the cellular-phone subscription rate and job-automatization risk, whereas Gnambs and Appel [17] identified a significant relation between the percentage of technological exports and geographical latitude. Despite the significance, these studies considered attitudes towards robots in the manufacturing context and a general appraisal of robots, respectively, and not specific attitudes towards robot for social services. Possible examples of the contextual variable that should be included are the percentage of older citizens and the unemployment rate, as employed by Gnambs and Appel [17]. However, other cultural indicators should also be included in future analyses.

The results also confirm $\mathrm{H} 2$, but only in relation to gender and town size. The effect of age and level of education were not significant, thus we cannot corroborate the results of previous studies present in the literature [17, 18, 40]. H2a was confirmed, and this means that moving from a low social class to a high social class generates a more positive attitude towards social robots. Furthermore, those living in towns are more likely to have a positive attitude than those living in rural areas. In light of these findings, we can affirm that individuals living in big cities and belonging to a high social class are more likely to indicate a favourable acceptance of robots for the provision of companionship. This finding is important as it infers that those belonging to a low social class, that is, with more social and economic disadvantages and who might stand to benefit from social robots the most, are in fact less likely to feel comfortable about receiving assistance from a robot.

The results do not support $\mathrm{H} 2 \mathrm{~b}$. Considering the employment variable, being a homemaker or retired but not a worker were associated with a greater level of acceptance towards social robots. In contrast with the findings reported by Gnambs and Appel [17], in which the employment condition was found to be significant, our study only found being a homemaker or retired to have a significant effect upon the dependent variable. However, it is important to bear in mind that a different variable "aggregation" was used to measure employment in the cited study, which referred to the same question adopted in the Eurobarometer questionnaire to collect information about employment. Gnambs and Appel [17] recoded it into three categories: 'white-collar workers', 'blue collar workers' and 'non-employed'. In the present analysis, different groups were used ('worker', 'homemaker', 'unemployed', 'retired' and 'still studying'). The information is substantively the same, but it is necessary to consider that the sample in the study by Gnambs and Appel [17] also includes data from the surveys carried out in the years 2012 and 2014, whereas the present work only considered the 2017 survey. The different effect of work condition on attitudes towards robots could thus be due to the fact that this study is underpowered compared with the research by Gnambs and Appel [17]. Similarly, education level was not found to influence the dependent variable in the present study, which previous research has, once again, found to have a significant relationship with opinions about robots $[17,18,40]$.

In summary, the analysis found that the main sociodemographic characteristics most associated with a positive attitude towards social robots for social assistance are being middle or high class, male and living with children in the city.

The results support $\mathrm{H} 2 \mathrm{c}$ since the indicators that regard technology confidence (in this case, AI knowledge) contributed towards having a positive opinion about robots in social services, as would be expected. Furthermore, all the TAM factors influencing motivation to adopt new technologies were significant. However, in the present study, digital technology efficacy had a negative impact, whereas in the study by Vu et al. [33] it had a positive effect. By contrast, the perceived threat of general job loss had a positive effect in the present study, but a negative one in the study by Vu et al. [33]. This could be due to the fact that the present research is specifically concerned with robots for companionship, whereas in that by $\mathrm{Vu}$ et al. [33] the dependent variable considers robots in general.

$\mathrm{H} 2$ also concerns the individual level predictor which states that people who use the internet frequently are more confident with robots (H2d). The analysis confirmed this to be true. People who use the internet daily are also more likely to express confident opinions about social robots than people who do not have any internet access. This finding should stimulate further reflections and research into the issues concerning the digital divide.

Finally, the results support $\mathrm{H} 3$, but they do not sustain $\mathrm{H}$, thus more different country level factors should be considered in further studies in order to identify which factors impact attitudes towards social robots at the contextual level. 
The purpose of this study was to answer the research questions using an approach that tests the hypotheses considering both the micro and the macro level. Although the results at the contextual level are not conclusive, the overall results provide important information for social policies makers, educators and organizations that will help them consolidate initiatives, formulate precise guidelines and best practices, and identify the most relevant variables to consider in order to facilitate the acceptance of social robots for the provision of companionship. Since our results show that people living under more disadvantaged conditions (i.e. belonging to a low social class and living in rural area) have less positive attitudes towards social robots, the initiatives to pursue at the educational, political and practical levels will require careful consideration in order to avoid that the use of the social robots for the assistance of the elderly generate more social inequality.

According to the informative statistics (AIC and BIC), the random slope model is the best model, and it shows a clear relationship between opinions about robots and having a positive attitude towards the use of robots for social services, a relationship which differs across countries.

The relatively small number of clusters (28 countries) could be considered a limitation of the study. Although the debate about the number of clusters to use remains open, various studies considering different numbers of level-2 units are present in the literature. Another possible limitation of the study could be the presence of non-significant variables, which could have been excluded from the statistical model, but were instead left in to demonstrate the model in its entirety.

Moreover, future studies should examine the effect of time in the evaluation of robots for social assistance since robots are expected to be increasingly introduced into this sphere of society [16]. Future work could also consider robot shape and specific functions. This research could be developed even further by considering the sub-national levels since national characteristics are not always homogeneous within any given country. This would also increase the number of the level-2 units and provide the opportunity to consider the countries again, even as a third level of analysis.

Acknowledgments I would like to thank Dr Andrew Bell from the University of Sheffield for introducing me to multilevel analysis during the Essex Summer School. I would also like to thank the anonymous Reviewers, whose comments helped improve this manuscript.

Funding This study received funding from the University of Verona Department of Human Sciences - [Grant ID: AdR3099/18 - "Progetto finanziato nell'ambito del programma Ricerca di Base 2017 promosso dall'Università degli Studi di Verona"]. Open access funding provided by Università degli Studi di Verona within the CRUI-CARE Agreement.

Open Access This article is licensed under a Creative Commons Attribution 4.0 International License, which permits use, sharing, adaptation, distribution and reproduction in any medium or format, as long as you give appropriate credit to the original author(s) and the source, provide a link to the Creative Commons licence, and indicate if changes were made. The images or other third party material in this article are included in the article's Creative Commons licence, unless indicated otherwise in a credit line to the material. If material is not included in the article's Creative Commons licence and your intended use is not permitted by statutory regulation or exceeds the permitted use, you will need to obtain permission directly from the copyright holder. To view a copy of this licence, visit http://creativecomm ons.org/licenses/by/4.0/.

\section{References}

1. Bogue R (2011) Robots in healthcare. Ind Robot: Int J 38(3):218-223. https://doi.org/10.1108/01439911111122699

2. Green T, Hartley N, Gillespie N (2016) Service provider's experiences of service separation. J Serv Res 19(4):477-494. https://doi. org/10.1177/1094670516666674

3. Robinson H, MacDonald B, Broadbent E (2014) The role of healthcare robots for older people at home: a review. Int J Soc Robot 6(4):575-591. https://doi.org/10.1007/s12369-014-0242-2

4. Fortunati L, Esposito A, Lugano G (2015) Introduction to the special issue "Beyond industrial robotics: social robots entering public and domestic spheres." Inf Soc 31(3):229-236. https://doi.org/10. 1080/01972243.2015.1020195

5. IFR Statistical Department (2009) Professional service robots are establishing themselves. In: World robotics 2009-service robots. IFR Statistical Department, Frankfurt. https://ifr.org/

6. Taipale S, de Luca F, Sarrica M, Fortunati L (2015) Robot shift from industrial production to social reproduction. In: Vincent $\mathrm{J}$, Taipale S, Sapio B, Lugano G, Fortunati L (eds) Social robots from a human perspective. Springer, Switzerland, pp 11-24

7. Young JE, Hawkins R, Sharlin E, Igarashi T (2009) Toward acceptable domestic robots: applying insights from social psychology. Int J Soc Robot 1(1):95-108. https://doi.org/10.1007/s12369-008-00 06-y

8. Broekens J, Heerink M, Rosendal H (2009) Assistive social robots in elderly care: a review. Gerontechnology 8(2):94-103. https:// doi.org/10.4017/gt.2009.08.02.002.00

9. Smarr CA, Mitzner TL, Beer JM, Prakash A, Chen TL, Kemp CC, Rogers WA (2014) Domestic robots for older adults: attitudes, preferences, and potential. Int J Soc Robot 6(2):229-247. https:// doi.org/10.1007/s12369-013-0220-0

10. Bodenhagen L, Suvei SD, Juel WK, Brander E, Krüger N (2019) Robot technology for future welfare: meeting upcoming societal challenges - an outlook with offset in the development in Scandinavia. Heal Technol 9(3):197-218. https://doi.org/10.1007/s1255 3-019-00302-x

11. Chen SC, Jones C, Moyle W (2018) Social robots for depression in older adults: a systematic review. J Nurs Scholarsh 50(6):612-622. https://doi.org/10.1111/jnu.12423

12. Bertolini A, Aiello G (2018) Robot companions: a legal and ethical analysis. Inf Soc 34(3):130-140. https://doi.org/10.1080/0197224 3.2018.1444249

13. Leenes R, Palmerini E, Koops BJ, Bertolini A, Salvini P, Lucivero F (2017) Regulatory challenges of robotics: some guidelines for addressing legal and ethical issues. Law Innov Technol 9(1):1-44. https://doi.org/10.1080/17579961.2017.1304921

14. Sparro R (2019) Robotics has a race problem. Sci Technol Hum Values 45(3):538-560. https://doi.org/10.1177/016224391986286 2

15. Vincent J, Taipale S, Sapio B, Lugano G, Fortunati L (eds) (2015) Social robots from a human perspective. Springer, Switzerland 
16. Shaw IGR (2017) Robot Wars: US Empire and geopolitics in the robotic age. Secur Dialogue 48(5):451-470. https://doi.org/10.11 77/0967010617713157

17. Gnambs T, Appel M (2019) Are robots becoming unpopular? Changes in attitudes towards autonomous robotic systems in Europe. Comput Hum Behav 93:53-61. https://doi.org/10.1016/ j.chb.2018.11.045

18. Hudson J, Orviska M, Hunady J (2017) People's attitudes to robots in caring for the elderly. Int J Soc Robot 9(2):199-210. https://doi. org/10.1007/s12369-016-0384-5

19. Naneva S, Sarda Gou M, Webb TL, Prescott TJ (2020) A systematic review of attitudes, anxiety, acceptance, and trust towards social robots. Int J Soc Robot. https://doi.org/10.1007/s12369-020-0065 9-4

20. Dahlin E (2019) Are robots stealing our jobs? Socius: Soc Res Dyn World 5:1-14. https://doi.org/10.1177/2378023119846249

21. Fortunati L (2013) Afterword: robot conceptualizations between continuity and innovation. Intervalla 1:116-129

22. Hegel F, Muhl C, Wrede B, Hielscher-Fastabend M, Sagerer G (2009) Understanding social robots. In: Conference: 2nd international conference on advances in computer-human interaction, ACHI 2009, February 1-7, 2009, Cancun, Mexico. https://doi.org/ 10.1109/ACHI.2009.51

23. Dautenhahn K, Billard A (1999) Bringing up robots or the psychology of socially intelligent robots. In: Proceedings of the 3rd annual conference on autonomous agents-AGENTS '99. https:// doi.org/10.1145/301136.301237

24. Pino M, Boulay M, Jouen F, Rigaud AS (2015) "Are we ready for robots that care for us?" Attitudes and opinions of older adults toward socially assistive robots. Front Aging Neurosci 7:141. https://doi.org/10.3389/fnagi.2015.00141

25. Policastro CA, Romero RAF, Zuliani G (2007) Robotic architecture inspired on behavior analysis. Int Joint Conf Neural Netw, Orlando, FL 2007:1482-1487. https://doi.org/10.1109/IJCNN.2007.437117 7

26. Baudrillard J (2012) Symbolic exchange and death. SAGE, Paris

27. Leite I, Martinho C, Paiva A (2013) Social robots for long-term interaction: a survey. Int J Soc Robot 5(2):291-308. https://doi. org/10.1007/s12369-013-0178-y

28. Wang N, Di Nuovo A, Cangelosi A, Jones R (2019) Temporal patterns in multi-modal social interaction between elderly users and service robot. Interact Stud 20(1):4-24. https://doi.org/10.107 5/is.18042.wan

29. Coghlan S (2021) Robots and the possibility of humanistic care. Int J Soc Robot. https://doi.org/10.1007/s12369-021-00804-7

30. Davis FD (1989) Perceived usefulness, perceived ease of use, and user acceptance of information technology. MIS Q 13(3):319-340

31. Ajzen I, Fishbein M (1980) Understanding attitudes and predicting social behavior. Prentice Hall, Englewood Cliffs

32. Huang MH, Rust RT (2018) Artificial intelligence in service. J Serv Res 21(2):155-172. https://doi.org/10.1177/1094670517752459

33. Vu HT, Lim J (2021) Effects of country and individual factors on public acceptance of artificial intelligence and robotics technologies: a multilevel SEM analysis of 28-country survey data. Behav Inf Technol. https://doi.org/10.1080/0144929X.2021.1884288
34. Vijayasarathy LR (2004) Predicting consumer intentions to use online shopping: the case for an augmented technology acceptance model. Inf Manag 41(6):747-762. https://doi.org/10.1016/j.im.20 03.08.011

35. Alsmadi HAD, Prybutok VR (2019) Factors that determine residents' acceptance of smart city technologies. Behav Inf Technol 39(6):610-623. https://doi.org/10.1080/0144929X.2019.1693629

36. Kelvyn J, Gould MI, Duncan C (2000) Death and deprivation: an exploratory analysis of deaths in the health and lifestyle survey. Soc Sci Med 50(7-8):1059-1079. https://doi.org/10.1016/s0277-9 536(99)00355-x (PMID: 10714927)

37. Katz JE, Halpern D, Crocker ET (2015) In the company of robots: views of acceptability of robots in social settings. In: Vincent $J$, Taipale S, Sapio B, Lugano G, Fortunati L (eds) Social robots from a human perspective. Springer, Switzerland, pp 24-38

38. Li D, Rau PPL, Li Y (2010) a cross-cultural study: effect of robot appearance and task. Int J Soc Robot 2(2):175-186. https://doi.org/ 10.1007/s12369-010-0056-9

39. Turja T, Oksanen A (2019) Robot acceptance at work: a multilevel analysis based on 27 EU countries. Int J Soc Robot 11(4):679-689. https://doi.org/10.1007/s12369-019-00526-X

40. Gnambs T (2019) (2019) Attitudes towards emergent autonomous robots in Austria and Germany. Elektrotech Inftech 136:296-300. https://doi.org/10.1007/s00502-019-00742-3

41. Snijders TAB, Bosker RJ (2012) Multilevel analysis. An introduction to basic and advanced multilevel modeling. Sage, London

42. Kelvyn J, Subramanian SV (2014) Developing multilevel models for analysing contextuality, heterogeneity and change using MlwiN, vol 1. University of Bristol, Bristol

43. Hox JJ (2010) Multilevel analysis: techniques and applications, 2nd edn. Routledge/Taylor \& Francis Group, New York

44. Leyland AH, Groenewegen PP (2020) Multilevel modelling and public health and health services research. Springer, Switzerland

45. Steele F (2008) Module 5: introduction to multilevel modelling (modelling concepts). http://www.bris.ac.uk/cmm/learning/ course.html

46. Blut M, Wang C (2020) Technology readiness: a meta-analysis of conceptualizations of the construct and its impact on technology usage. J Acad Mark Sci 48:649-669. https://doi.org/10.1007/s117 47-019-00680-8

Publisher's Note Springer Nature remains neutral with regard to jurisdictional claims in published maps and institutional affiliations.

Marco Carradore is a lecturer in the Department of Cultures and Civilizations and in the School of Science and Engineering at the University of Verona. He holds an M.Sc. in Social Science Data Analysis from the University of Essex and a Ph.D. in Sociology and Social Research from Verona University. His research interests include Socially Assistive Robots and the impact that the technological artefacts have on welfare development. 